\title{
Differential equations with generalized coefficients
}

\author{
Aleh Yablonski \\ Department of Functional Analysis, Belarusian State University, \\ F.Skaryna av.,4, 220050, Minsk, BELARUS \\ Email: yablonski@bsu.by \\ Department of Mathematics, University of Oslo \\ Box 1053 Blindern, N-0316 Oslo, NORWAY, \\ Email: alehy@math.uio.no
}

\begin{abstract}
Differential equations with generalized coefficients by using the algebra of new generalized functions are investigated. It is shown that the different interpretations of the solutions of such equations can be described by the unique approach of the algebra of new generalized functions.
\end{abstract}

Key words and phrases: algebra of new generalized functions, differential equations with generalized coefficients, functions of finite variation.

2000 Mathematics subject classification: 34A37, 34K45, 46F30.

\section{Introduction}

The theory of generalized functions is one of the most powerful tools for investigating linear differential equations. However, from the very beginning the distribution theory has an essential disadvantage: it is inapplicable to the solution of nonlinear problems. Therefore different interpretations of the solution of the nonlinear differential equations were proposed by many mathematicians. Unfortunately different interpretations of the same equation lead, in general, to different solutions. See, e.g., [1, 2, 3, 4, 5, 6, 7]. Usually differential equations are used to describe the dynamics of real systems or phenomena. In order to choose adequate interpretations of such equations one has to consider the reasons which were used for modeling the dynamics of the real systems by these equations.

In this paper we will consider the following nonlinear equation with generalized coefficients

$$
\dot{X}(t)=f(X(t)) \dot{L}(t)+g(X(t)),
$$

where $t \in[a ; b] \subset \mathbb{R}, \dot{L}(t)$ is a derivative of the function of finite variation in the distributional sense. In general, since $\dot{L}(t)$ is a distribution and the function $f(X(t))$ is not smooth then the product $f(X(t)) \dot{L}(t)$ is not well defined and the solution of the equation (1.1) essentially depends on the interpretation. Recall some approaches to the interpretation of the equation $(1.1)$. 
The first approach is concerned with considering the equation in the framework of distribution theory. According to this approach the product of distributions from some classes are defined and then one tries to find the solution of the equation (1.1) in these classes of distributions. For example in the papers $[1,4,5]$ the product of some distributions and discontinuous functions was defined. See also monograph [7, Ch. 1, §8] for another definition. Notice that the solutions of the equation (1.1) which can be obtained by using the products from $[1,4,5]$ and [7] are different.

The second approach is to interpret the equation (1.1) as the following integral equation

$$
x(t)=x^{0}+\int_{a}^{t} f(x(s)) d L(s)+\int_{a}^{t} g(s) d s,
$$

where the integrals are understood in Lebesgue-Stieltjes, Perron-Stieltjes, etc., sense. See, e.g., $[2,6]$. But in this approach the solution of the integral equation depends on the interpretation of the integral and the definition of the function $x(t)$ in the discontinuity points of $L(t)$.

The third approach is based on the idea of the approximation of the solution of the equation (1.1) by the solutions of the ordinary differential equations, which are constructed by using the smooth approximation of the function $L(t)$. In the monograph [7, Ch. 4] it was shown that in this case the limit of the solutions of the smoothed equations exists; it is called "approximative solution" and it is a solution of the following equation

$$
x(t)=x^{0}+\int_{a}^{t} f(x(s)) d L^{c}(s)+\int_{a}^{t} g(x(s)) d s+\sum_{\zeta_{i}<t} S\left(\zeta_{i}, x\left(\zeta_{i}-\right), \Delta L\left(\zeta_{i}\right)\right),
$$

where $L^{c}(t)$ is a continuous part of the function $L(t), \zeta_{i}$ and $\Delta L\left(\zeta_{i}\right)=L\left(\zeta_{i}+\right)-L\left(\zeta_{i}-\right)$ are the epochs and the sizes of jumps of the function $L$ respectively, and the function $S\left(\zeta_{i}, x\left(\zeta_{i}-\right), \Delta L\left(\zeta_{i}\right)\right)$ is defined as a solution of some auxiliary ordinary differential equation. Notice that the solution of the last equation coincides with the solution of the equation (1.1) which was obtained in [7] by using the definition of the product of distributions.

In this paper we will consider the equation (1.1) by using the algebra of new generalized functions from [8]. This is to say we will interpret the equation (1.1) as the equation in the differentials in the algebra of new generalized functions. Such interpretation says that the solution of the equation (1.1) is a new generalized function.

The main purpose of the article is to show that under some conditions this new generalized function associates with some ordinary function which is natural to call the solution of the equation (1.1). Also it will be shown that the solutions of the equation (1.1) in the sense of the previous approaches can be obtained from the solution of the equation in the differentials in the algebra of new generalized functions.

The method of analogous algebra of new generalized stochastic processes was successfully used in the articles $[9,10,11]$ for researching stochastic differential equations.

\section{The algebra of new generalized functions}

In this section we recall the definition of the algebra of new generalized functions from [8].

At first we define an extended real line $\widetilde{\mathbb{R}}$ using a construction typical for non-standard analysis. 
Let $\overline{\mathbb{R}}=\left\{\left(x_{n}\right)_{n=1}^{\infty}: x_{n} \in \mathbb{R}\right.$ for all $\left.n \in \mathbb{N}\right\}$ be a set of real sequences. We will call two sequences $\left\{x_{n}\right\} \in \overline{\mathbb{R}}$ and $\left\{y_{n}\right\} \in \overline{\mathbb{R}}$ equivalent if there is a natural number $N$ such that $x_{n}=y_{n}$ for all $n>N$.

The set $\widetilde{\mathbb{R}}$ of equivalence classes will be called the extended real line and any of the classes a generalized real number.

It is easy to see that $\mathbb{R} \subset \widetilde{\mathbb{R}}$ because one may associate with any ordinary number $x \in \mathbb{R}$ a class containing a stationary sequence with $x_{n}=x$. It is evident that $\widetilde{\mathbb{R}}$ is an algebra. The product $\widetilde{x} \widetilde{y}$ of two generalized real numbers is defined as the class of sequences equivalent to the sequence $\left\{x_{n} y_{n}\right\}$, where $\left\{x_{n}\right\}$ and $\left\{y_{n}\right\}$ are the arbitrary representatives of the classes $\widetilde{x}$ and $\widetilde{y}$ respectively.

For any segment $\mathbf{T}=[a ; b] \subset \mathbb{R}$ one can construct an extended segment $\widetilde{\mathbf{T}}$ in a similar way. Let $H$ denote the subset of $\widetilde{\mathbb{R}}$ of nonnegative "infinitely small numbers": $H=\{\widetilde{h} \in$ $\left.\widetilde{\mathbb{R}}: \widetilde{h}=\left[\left\{h_{n}\right\}\right], h_{n}>0, \lim h_{n}=0\right\}$.

Consider the set of sequences of infinitely differentiable functions $\left\{f_{n}(x)\right\}$ on $\mathbb{R}$. We will call two sequences $\left\{f_{n}(x)\right\}$ and $\left\{g_{n}(x)\right\}$ equivalent if for each compact set $K \subset \mathbb{R}$ there is a natural number $N$ such that $f_{n}(x)=g_{n}(x)$ for all $n>N$ and $x \in K$. The set of classes of equivalent functions is denoted by $\mathcal{G}(\mathbb{R})$ and its elements are called new generalized functions. Similarly one can define the space $\mathcal{G}(\mathbf{T})$ for any interval $\mathbf{T}=[a ; b]$.

For each distribution $f$ we can construct a sequence $f_{n}$ of smooth functions such that $f_{n}$ converges to $f$ (e.g. one can consider the convolution of $f$ with some $\delta$-sequence). This sequence defines the new generalized function which corresponds to the distribution $f$. Thus the space of distributions is a subset of the algebra of new generalized functions. However, in this case, infinitely many new generalized functions correspond to one distribution (e.g., by taking different $\delta$-sequences). We will say that the new generalized function $\tilde{f}=\left[\left\{f_{n}\right\}\right]$ associates with the ordinary function or distribution $f$ if $f_{n}$ converges to $f$ in some sense.

Let $\widetilde{f}=\left[\left\{f_{n}(x)\right\}\right]$ and $\widetilde{g}=\left[\left\{g_{n}(x)\right\}\right]$ be generalized functions. Then there is defined a composition $\widetilde{f} \circ \widetilde{g}=\left[\left\{f_{n}\left(g_{n}(x)\right)\right\}\right] \in \mathcal{G}(\mathbb{R})$. In the same way one can define the value of the new generalized function $\widetilde{f}$ at the generalized real point $\widetilde{x}=\left[\left\{x_{n}\right\}\right] \in \widetilde{\mathbb{R}}$ as $\widetilde{f}(\widetilde{x})=\left[\left\{f_{n}\left(x_{n}\right)\right\}\right]$.

For each $\widetilde{h}=\left[\left\{h_{n}\right\}\right] \in H$ and $\widetilde{f}=\left[\left\{f_{n}(x)\right\}\right] \in \mathcal{G}(\mathbb{R})$ we define a differential $d_{\widetilde{h}} \widetilde{f} \in \mathcal{G}(\mathbb{R})$ by $d_{\widetilde{h}} \tilde{f}=\left[\left\{f_{n}\left(x+h_{n}\right)-f_{n}(x)\right\}\right]$. The construction of the differential was proposed by Lazakovich (see $[12,9]$ ) for the algebra of new generalized stochastic processes.

Now we can give an interpretation of the equation (1.1) using the methods of the introduced algebras $\mathcal{G}(\mathbb{R})$ and $\mathcal{G}(\mathbf{T})$. Let $L(t), t \in[a ; b]=\mathbf{T}$ be a right-continuous function of finite variation. We replace ordinary functions in equation (1.1) by corresponding new generalized functions and then write the algebra's differentials. So we have

$$
d_{\widetilde{h}} \widetilde{X}(\widetilde{t})=\widetilde{f}(\widetilde{X}(\widetilde{t})) d_{\widetilde{h}} \widetilde{L}(\widetilde{t})+\widetilde{g}(\widetilde{X}(\widetilde{t})) d_{\widetilde{h}} \widetilde{t}
$$

with initial value $\left.\widetilde{X}\right|_{[\widetilde{a} ; \widetilde{h})}=\widetilde{X}^{0}$, where $\widetilde{h}=\left[\left\{h_{n}\right\}\right] \in H, \widetilde{a}=[\{a\}] \in \widetilde{\mathbf{T}}, \widetilde{t}=\left[\left\{t_{n}\right\}\right] \in \widetilde{\mathbf{T}}$, $\widetilde{X}=\left[\left\{X_{n}(t)\right\}\right], \widetilde{f}=\left[\left\{f_{n}(x)\right\}\right], \widetilde{g}=\left[\left\{g_{n}(x)\right\}\right], \widetilde{X}^{0}=\left[\left\{X_{n}^{0}(t)\right\}\right], \widetilde{L}=\left[\left\{L_{n}(t)\right\}\right]$, and $L_{n} \rightarrow L$, $b_{n} \rightarrow b, \sigma_{n} \rightarrow \sigma$ and $X_{n}^{0} \rightarrow X(0)$. If $\widetilde{X}$ associates with some (generalized) function $X$ then we say that $X$ is a solution of the equation (1.1).

The purpose of the present paper is to investigate when the solution $\widetilde{X}$ of the equation (2.1) associates with some ordinary function and to describe possible associated solutions. 


\section{Main results}

In this section we will formulate the main theorem. The proof of the theorem will be given in the next sections.

Let $L(t), t \in \mathbf{T}=[a ; b]$ be a right-continuous function of finite variation and $L(a)=0$. We will assume that $L(t)=L(b)$ if $t>b$ and $L(t)=L(a)$ if $t<a$. Denote by $\mathrm{V}_{u}^{v} L$ the total variation of the function $L$ on the interval $[u ; v] \subset \mathbf{T}$. Suppose that $f$ and $g$ are Lipschitz continuous with constant $M$ and for all $x \in \mathbb{R}$

$$
|f(x)|+|g(x)| \leq M(1+|x|) .
$$

Consider as representatives of the new generalized functions $\widetilde{f}, \widetilde{g}$ and $\widetilde{L}$ from the equation (2.1) the following convolutions with $\delta$-sequence:

$$
L_{n}(t)=\left(L * \rho_{n}\right)(t)=\int_{0}^{1 / n} L(t+s) \rho_{n}(s) d s, g_{n}=g * \rho_{n}, f_{n}=f * \rho_{n},
$$

where $\rho_{n} \in C^{\infty}(\mathbb{R}), \rho_{n} \geq 0$, supp $\rho_{n} \subseteq[0 ; 1 / n]$ and $\int_{0}^{1 / n} \rho_{n}(s) d s=1$.

By using the representatives we can rewrite the equation (2.1) in the following form:

$$
\left\{\begin{array}{l}
X_{n}\left(t+h_{n}\right)-X_{n}(t)=f_{n}\left(X_{n}(t)\right)\left(L_{n}\left(t+h_{n}\right)-L_{n}(t)\right)+g_{n}\left(X_{n}(t)\right) h_{n}, \\
\left.X_{n}\right|_{\left[a ; a+h_{n}\right)}(t)=X_{n}^{0}(t) .
\end{array}\right.
$$

The solution $\tilde{X}$ of the equation (2.1) associates with some function if and only if the sequence of the solutions $X_{n}$ of the equation (3.3) converges. Therefore we have to investigate the limiting behavior of the sequence $X_{n}$.

Let $t$ be an arbitrary point of $\mathbf{T}$. There exists $m_{t} \in \mathbb{N}$ and $\tau_{t} \in\left[a ; a+h_{n}\right)$ such that $t=\tau_{t}+m_{t} h_{n}$. Set $t_{k}=\tau_{t}+k h_{n}, k=0,1, \ldots, m_{t}$. Then the solution of the equation (3.3) can be written as

$$
X_{n}(t)=X_{n}^{0}\left(\tau_{t}\right)+\sum_{k=0}^{m_{t}-1} f_{n}\left(X_{n}\left(t_{k}\right)\right)\left(L_{n}\left(t_{k+1}\right)-L_{n}\left(t_{k}\right)\right)+\sum_{k=0}^{m_{t}-1} g_{n}\left(X_{n}\left(t_{k}\right)\right) h_{n} .
$$

Consider the function $F_{n}(x):[-\infty ;+\infty] \rightarrow[0 ; 1]$ given by

$$
F_{n}(x)=\int_{x}^{1 / n} \rho_{n}(s) d s
$$

Since $\rho_{n}(s) \geq 0$, then $F_{n}$ is a non-increasing function, $0 \leq F_{n}(x) \leq 1$ and $F_{n}(+\infty)=0$, $F_{n}(-\infty)=1$. Denote by $F_{n}^{-1}$ the inverse function of $F_{n}$, i.e., $F_{n}^{-1}:[0 ; 1] \rightarrow[-\infty ;+\infty]$ and

$$
F_{n}^{-1}(u)=\sup \left\{x: F_{n}(x)=u\right\} .
$$

In order to describe the limits of the sequence $X_{n}$ we consider the integral equation

$$
\begin{aligned}
& X(t)=x^{0}+\int_{a}^{t} g(X(s)) d s+\int_{a}^{t} f(X(s)) d L^{c}(s) \\
& +\sum_{a<s \leq t}(\varphi(\Delta L(s) f, X(s-), 1)-X(s-)), t \in \mathbf{T},
\end{aligned}
$$


where $L^{c}$ is a continuous part of the function $L, \Delta L(s)=L(s+)-L(s-)$ is a size of the jump of the function $L$ at the epoch $s$, and $\varphi(z, x, u)$ denotes the solution of the integral equation

$$
\varphi(z, x, u)=x+\int_{[0 ; u)} z(\varphi(z, x, v)) \mu(d v),
$$

and $\mu(d u)$ is a probability measure defined on the Borel subsets of the interval $[0 ; 1]$. In order to underline the dependence $\varphi(z, x, u)$ on the measure $\mu$ sometimes we will also use the notation $\varphi(z, x, u, \mu)$. Here and in what follows all integrals are understood in the Lebesgue-Stieltjes sense.

Remark 3.1 Let us note some particular cases of the equation (3.7).

If the measure $\mu$ gives the mass one to the point 0 , then the equation (3.8) has the solution

$$
\varphi(z, x, u)=\left\{\begin{array}{l}
x, u=0 \\
x+z(x), u \in(0 ; 1]
\end{array}\right.
$$

Hence the equation (3.7) becomes

$$
X(t)=x^{0}+\int_{(a, t]} f(X(s-)) d L(s)+\int_{a}^{t} g(X(s)) d s .
$$

The interpretation of the equation (1.1) as the equation (3.9) was considered by Das and Sharma [2], see also Pandit and Deo [6].

If the measure $\mu$ is the Lebesgue measure, then the equation (3.8) can be written as an ordinary differential equation

$$
\frac{\partial \varphi(z, x, u)}{\partial u}=z(\varphi(z, x, u))
$$

and $\varphi(z, x, 0)=x$. Then the solution of the equation (3.7) is a so-called "approximative solution" (see, e.g. [7]). It was shown by Zavalishchin and Sesekin in [7] that if we consider approximation $L_{n}^{\prime}$ of $L$, then the sequence $X_{n}^{\prime}$ of the solutions of the equation (1.1), where $L$ is replaced by $L_{n}^{\prime}$ converges to the "approximative solution", i.e., to the solution of the equation (3.7) with the measure $\mu$ equal to the Lebesgue measure.

The solution of the equation in the sense of the papers [1, 4, 5] can be obtained from the equation (3.7) by using appropriate measure $\mu$.

The equation (3.8) has a unique solution if, for example, the function $z$ is Lipschitz continuous (see, e.g. [13]). Since we concerned with the case where $f$ is globally Lipschitz, then the function $\varphi(\Delta L(s) f, X(s-), 1)$ is always defined.

We shall discuss the existence and some properties of the solutions of the equations (3.7) and (3.8) in Section 4.

Definition 3.2 We say that the function $\sigma:[0 ; 1] \rightarrow[0 ; 1]$ belongs to class $\mathbf{G}$ if there is a system of pairwise-disjoint intervals $\left(a_{i} ; b_{i}\right) \subseteq[0 ; 1], i \in I$ such that

$$
\sigma(u)=\left\{\begin{array}{l}
b_{i}, u \in\left(a_{i} ; b_{i}\right] \\
u, u \notin \bigcup_{i \in I}\left(a_{i} ; b_{i}\right]
\end{array}\right.
$$


Remark 3.3 There are two most important examples of the functions from the class $\mathbf{G}$ :

1. $\sigma(u)=\mathbf{1}_{(0 ; 1]}(u)$, i.e. $\sigma(u)=1$ if $u>0$ and $\sigma(u)=0$ if $u=0$. In this case $I=\{1\}$ and $\left(a_{1} ; b_{1}\right]=(0 ; 1]$. The measure $\mu$ generated by this function gives the mass one to the point 0 , and it follows from Remark 3.1 that the equation (3.7) with this measure $\mu$ can be written in the form (3.9).

2. $\sigma(u)=u$. In this case $I=\emptyset$. From Remark 3.1 we deduce that the solution of the equation (3.7) with the measure generated by $\sigma$ is an "approximative solution".

The following theorem describes the limits of the sequence $X_{n}$.

Theorem 3.4 Let $f$ and $g$ be Lipschitz functions satisfying (3.1), $\int_{t \in \mathbf{T}}\left|X_{n}^{0}\left(\tau_{t}\right)-x^{0}\right| d t \rightarrow 0$ and $F_{n}\left(F_{n}^{-1}(u)-\delta h_{n}\right) \rightarrow \sigma(u)$ as $n \rightarrow \infty$ and $h_{n} \rightarrow 0$ for all $\delta \in(0 ; 1)$ and for any continuity point $u \in[0 ; 1]$ of $\sigma$. Then $\sigma$ belongs to class $\mathbf{G}$ and

$$
\int_{\mathbf{T}}\left|X_{n}(t)-X(t)\right| d t \rightarrow 0
$$

as $n \rightarrow \infty$ and $h_{n} \rightarrow 0$, where $X(t)$ is a solution of the equation (3.7) with the measure $\mu$ generated by the function $\sigma$.

If $\delta$-sequence $\rho_{n}$ has the form $\rho_{n}(u)=n \rho(n u)$, where $\rho \in C^{\infty}(\mathbb{R}), \rho \geq 0$, supp $\rho \subseteq[0 ; 1]$ and $\int_{0}^{1} \rho(u) d u=1$, then we will say that $\delta$-sequence $\rho_{n}$ is of the simplest type.

Corollary 3.5 Let $\delta$-sequence $\rho_{n}$ be of the simplest type. Then the sequence $F_{n}\left(F_{n}^{-1}(u)-\right.$ $\left.\delta h_{n}\right)$ converges weakly for any $\delta \in(0 ; 1)$ and the limit does not depend on $\delta$ if and only if either $1 / n=o\left(h_{n}\right)$ or $h_{n}=o(1 / n)$. Moreover, suppose that $f$ and $g$ are global Lipschitz functions and $\int_{t \in \mathbf{T}}\left|X_{n}^{0}\left(\tau_{t}\right)-x^{0}\right| d t \rightarrow 0$ as $n \rightarrow \infty$ and $h_{n} \rightarrow 0$. Then

$$
\int_{\mathbf{T}}\left|X_{n}(t)-X(t)\right| d t \rightarrow 0
$$

where $X_{n}$ is a solution of the equation (3.3) and

1. $X(t)$ is a solution of the equation (3.9) if $1 / n=o\left(h_{n}\right)$,

2. $X(t)$ is a solution of the equation (3.7) with the measure $\mu$ is equal to the Lebesgue measure, if $h_{n}=o(1 / n)$.

Notice that $F_{n}\left(F_{n}^{-1}(u)-\delta h_{n}\right) \rightarrow \mathbf{1}_{(0 ; 1]}(u)$ if and only if $1 / n=o\left(h_{n}\right)$. And $F_{n}\left(F_{n}^{-1}(u)-\right.$ $\left.\delta h_{n}\right) \rightarrow u$ if and only if $h_{n}=o(1 / n)$. Hence Remark 3.3 and Corollary 3.5 imply that for the $\delta$-sequence of the simplest type the solutions $X_{n}$ of the equation (3.3) converge either to the solution of the equation (1.1) in the sense of papers $[2,6]$ if $1 / n=o\left(h_{n}\right)$ or to the "approximative solution" of the equation (1.1) in the sense of monograph [7] if $h_{n}=o(1 / n)$.

For a continuous function $L$ equation (3.7) has not the last term, and hence is equivalent to the equation (3.9). Therefore we have the following result

Corollary 3.6 Let $L$ be a continuous function of finite variation on $\mathbf{T}$. Suppose that $f$ and $g$ are Lipschitz continuous and $\int_{t \in \mathbf{T}}\left|X_{n}^{0}\left(\tau_{t}\right)-x^{0}\right| d t \rightarrow 0$ as $n \rightarrow \infty$ and $h_{n} \rightarrow 0$. Then

$$
\int_{\mathbf{T}}\left|X_{n}(t)-X(t)\right| d t \rightarrow 0
$$

where $X_{n}$ is a solution of the equation (3.3), and $X(t)$ is a solution of the equation (3.9).

Remark 3.7 It will follow from the proof of Theorem 3.4 that $L^{1}$-norm can be replaced by sup-norm if the initial condition $X_{n}^{0}$ converges in sup-norm. 


\section{Discussing of the equations}

In this section we consider the equation (3.7) and show that it has the unique solution. We will prove also some inequalities for solutions of the equations (3.7) and (3.8).

The following modification of Gronwall's inequality from [13] will be very useful in the sequel.

Lemma 4.1 ([13]) Let $\nu$ be a locally bounded measure on $\mathbb{R}$, and let $Y(t)$, $t \geq 0$ be a function of bounded variation on each bounded interval such that for all $t \geq 0$

$$
0 \leq Y(t) \leq A+B \int_{[0 ; t)} Y(s) \nu(d s)
$$

where $A$ and $B$ are nonnegative constants.

Then for each $t \geq 0$

$$
Y(t) \leq A e^{B \nu([0 ; t))} \prod_{s<t}(1+B \nu(\{s\})) e^{-B \nu(\{s\})} \leq A e^{B \nu([0 ; t))} .
$$

We will also need the stronger inequality in the discrete case (see, [14]).

Lemma $4.2([\mathbf{1 4}])$ Let $a_{n}, b_{n}$ and $y_{n}(n=1,2, \ldots)$ are nonnegative real numbers and if $x_{n} \leq a_{n}+\sum_{i=1}^{n-1} b_{i} x_{i}$ for all $n=1,2, \ldots$, then

$$
x_{n} \leq a_{n}+\frac{1}{p_{n-1}} \sum_{i=1}^{n-1} a_{i} b_{i} p_{i} \leq \max _{1 \leq i \leq n} a_{i} e^{\sum_{j=1}^{n-1} b_{j}}
$$

for all $n=1,2, \ldots$, where $p_{k}=\prod_{i=1}^{k}\left(1+b_{i}\right)^{-1} \quad(k=1,2, \ldots)$.

By using Lemma 4.1 it is easy to show from the definition of $\varphi(z, x, u)$ the following inequalities.

Lemma 4.3 Let $\varphi(z, x, u)$ be a solution of the equation (3.8) with real-valued function $z$ such that $|z(x)-z(y)| \leq K|x-y|$ and $|z(x)| \leq K(1+|x|)$ for all $x, y \in \mathbb{R}$. Then the following inequalities hold for all $x, y \in \mathbb{R}, u, v \in[0 ; 1], u>v$ :

1. $|\varphi(z, x, u)-\varphi(z, y, u)| \leq|x-y| e^{K}$.

2. $|\varphi(z, x, u)| \leq(K+|x|) e^{K}$.

3. $|\varphi(z, x, u)-x| \leq K\left(1+(|x|+K) e^{K}\right)$.

4. $|\varphi(z, x, u)-x-\varphi(z, y, u)+y| \leq|x-y| K e^{K}$.

5. $|\varphi(z, x, u)-\varphi(z, x, v)| \leq K\left(1+(K+|x|) e^{K}\right) \mu([v, u))$

For a given function of finite variation $L$, we define

$$
h(s, x)=\frac{\varphi(\Delta L(s) f, x, 1)-x}{\Delta L(s)}=\int_{[0 ; 1)} f(\varphi(\Delta L(s) f, x, u)) \mu(d u) .
$$


By using this function we can rewrite the equation (3.7) in the following form:

$$
\begin{aligned}
X(t)= & x^{0}+\int_{a}^{t} g(X(s)) d s+\int_{a}^{t} f(X(s)) d L^{c}(s) \\
& +\int_{(a ; t]} h(s, X(s-)) d L^{d}(s), t \in \mathbf{T},
\end{aligned}
$$

where $L^{d}$ is the discontinuous part of the function $L$, i.e. $L^{d}=L-L^{c}$.

Since $\varphi(0, x, u)=x$ for all $x \in \mathbb{R}$ and $u \in[0 ; 1]$, then $h(s, x)=f(x)$ if $\Delta L(s)=0$ and the equation (4.1) can be written as follows:

$$
X(t)=x^{0}+\int_{a}^{t} g(X(s)) d s+\int_{(a ; t]} h(s, X(s-)) d L(s), t \in \mathbf{T} .
$$

Lemma 4.4 For $f$ Lipschitz continuous with constant $M$, the following inequality holds:

$$
|h(s, x)-h(s, y)| \leq M|x-y| e^{M \Delta L(s)} .
$$

Proof. From the definitions of the functions $h$ and $\varphi$ we have

$$
\begin{aligned}
& |h(s, x)-h(s, y)| \leq \int_{[0 ; 1)}|f(\varphi(\Delta L(s) f, x, v))-f(\varphi(\Delta L(s) f, y, v))| \mu(d v) \\
& \leq M \int_{[0 ; 1)}|\varphi(\Delta L(s) f, x, v)-\varphi(\Delta L(s) f, y, v)| \mu(d v) \leq M|x-y| e^{M \Delta L(s)},
\end{aligned}
$$

where we have used Lemma 4.3 for the last inequality.

It was shown by Groh [13] that the homogeneous equation (4.2), i.e., $g=0$, has the unique solution for a Lipschitz continuous function $h$. Since by Lemma 4.4 the function $h$ satisfies a uniform Lipschitz condition provided $f$ is Lipschitz continuous, then one can use an evident modification of the method proposed in [13] in order to prove the existence and the uniqueness of the solution of the equation (4.2) if $g$ is Lipschitz continuous, too. Since the equations (4.2) and (3.7) are equivalent, then the equation (3.7) has a unique solution.

Lemma 4.5 Let functions $f$ and $g$ be Lipschitz continuous with constant $M$ satisfying (3.1). Then for solutions $X$ and $X_{n}$ of the equations (3.7) and (3.3), respectively, the following inequalities hold for all $t, s \in \mathbf{T}, t>s$ and $l, n \in \mathbb{N}$ :

1. $|X(t)| \leq C\left(1+\left|x^{0}\right|\right)$;

$$
\left|X_{n}(t)\right| \leq C\left(1+\left|X_{n}^{0}\left(\tau_{t}\right)\right|\right) \text {, }
$$

where the constant $C$ depends only on $M,|\mathbf{T}|$ and $\mathrm{V}_{a}^{b} L$.

2. $|X(t)-X(s)| \leq M\left(1+C\left(1+\left|x^{0}\right|\right)\right)\left((t-s)+\mathrm{V}_{s+}^{t} L\right)$.

3. $\left|X_{n}\left(t+l h_{n}\right)-X_{n}(t)\right| \leq M\left(1+C\left(1+\left|X_{n}^{0}\left(\tau_{t}\right)\right|\right)\right)\left(l h_{n}+\mathrm{V}_{t}^{t+l h_{n}} L_{n}\right) \leq M(1+C(1+$ $\left.\left.\left|X_{n}^{0}\left(\tau_{t}\right)\right|\right)\right)\left(l h_{n}+\mathrm{V}_{t+}^{t+l h_{n}+1 / n} L\right)$.

Proof. The proof of these inequalities is standard and it uses the definitions of $X$ and $X_{n}$, Lemma 4.1, inequality (3.1), and Lipschitz continuity of $f$ and $g$. 


\section{$5 \quad$ Auxiliary statements}

Let us introduce some notations. The function of bounded variation $L$ admits the following unique representation $L=L^{c}+L^{d}$, where $L^{c}$ is a continuous function and $L^{d}$ piecewise constant function. Since $L$ is a right continuous function, then $L^{d}$ is a right continuous, too. Denote by $\zeta_{i}, i \in \mathbb{N}$ the epochs of jumps of the function $L$. It is evident, that $L^{d}(t)=\sum_{\zeta_{i} \leq t} \Delta L\left(\zeta_{i}\right)$. Moreover $\mathrm{V}_{u}^{v} L=\mathrm{V}_{u}^{v} L^{c}+\mathrm{V}_{u}^{v} L^{d}$ for any $a \leq u<v \leq b$ and $\mathrm{V}_{u}^{v} L^{d}=$ $\sum_{u \leq \zeta_{i} \leq v}\left|\Delta L\left(\zeta_{i}\right)\right|$. We assume that if $L$ has only $q$ jumps, then $\zeta_{q+1}=\zeta_{q+2}=\cdots=b+1$ and $\Delta L\left(\zeta_{q+1}\right)=\Delta L\left(\zeta_{q+2}\right)=\cdots=0$

Fix an arbitrary $\varepsilon>0$. Since $\bigvee_{a}^{b} L<\infty$, then $\bigvee_{a}^{b} L^{d}=\sum_{i=1}^{\infty}\left|\Delta L\left(\zeta_{i}\right)\right|<\infty$. Hence there is a number $N \in \mathbb{N}$ such that $\sum_{i=N+1}^{\infty}\left|\Delta L\left(\zeta_{i}\right)\right| \leq \varepsilon$. We can represent $L^{d}$ in the following way:

$$
L^{d}=L^{\leq N}+L^{>N},
$$

where $L^{\leq N}(t)=\sum_{i=1}^{N} \mathbf{1}_{\left\{\zeta_{i} \leq t\right\}} \Delta L\left(\zeta_{i}\right)$ and $L^{>N}(t)=\sum_{i=N+1}^{\infty} \mathbf{1}_{\left\{\zeta_{i} \leq t\right\}} \Delta L\left(\zeta_{i}\right)$. From this it follows readily that $\mathrm{V}_{u}^{v} L^{>N} \leq \varepsilon$ and $\mathrm{V}_{u}^{v} L^{\leq N} \leq \mathrm{V}_{u}^{v} L^{d}<\infty$ for all $a \leq u<v \leq b$.

Set $L_{n}^{c}=L^{c} * \rho_{n}, L_{n}^{d}=L^{d} * \rho_{n}, L_{n}^{\leq N}=L^{\leq N} * \rho_{n}$ and $L_{n}^{>N}=L^{>N} * \rho_{n}$, where $\rho_{n}$ from the formula (3.2). Then we have $L_{n}=L_{n}^{c}+L_{n}^{d}$ and $L_{n}^{d}=L_{n}^{\leq N}+L_{n}^{>N}$. Furthermore for all $t \in \mathbf{T}$ we have

$$
\left|L_{n}^{c}(t)-L^{c}(t)\right| \leq \mathrm{V}_{t}^{t+1 / n} L^{c} .
$$

From now on we will denote by $C$ the constant which does not depend on $n, h_{n}$ and $t \in \mathbf{T}$, and its value can change from one formula to another.

Lemma 5.1 Suppose that $f$ and $g$ are Lipschitz continuous functions. Let $X_{n}(t)$ and $X(t)$ be solutions of the equations (3.3) and (3.7) respectively. Then for all $t \in \mathbf{T}$

$$
\begin{gathered}
\left|\sum_{k=0}^{m_{t}-1} g_{n}\left(X_{n}\left(t_{k}\right)\right) h_{n}-\int_{a}^{t} g(X(s)) d s\right| \leq \\
C h_{n}+C / n+C h_{n} \sum_{k=0}^{m_{t}-1}\left|X_{n}\left(t_{k}\right)-X\left(t_{k}\right)\right| .
\end{gathered}
$$

Proof. The proof of the lemma is standard and, therefore, is omitted.

Lemma 5.2 Suppose that $f$ and $g$ are Lipschitz continuous functions. Let $X_{n}(t)$ and $X(t)$ be solutions of the equations (3.3) and (3.7) respectively. Then for all $t \in \mathbf{T}$

$$
\begin{gathered}
\left|\sum_{k=0}^{m_{t}-1} f_{n}\left(X_{n}\left(t_{k}\right)\right)\left(L_{n}^{c}\left(t_{k+1}\right)-L_{n}^{c}\left(t_{k}\right)\right)-\int_{a}^{t} f(X(s)) d L^{c}(s)\right| \leq C h_{n}+C / n \\
+C\left(1+\left|X_{n}^{0}\left(\tau_{t}\right)\right|\right) \sup _{|u-v| \leq h_{n}+1 / n} \mathrm{~V}_{u}^{v} L^{c}+C \sum_{k=0}^{m_{t}-1}\left|X_{n}\left(t_{k}\right)-X\left(t_{k}\right)\right|\left|L^{c}\left(t_{k+1}\right)-L^{c}\left(t_{k}\right)\right| .
\end{gathered}
$$


ProOF. The following representation is evident.

$$
\begin{gathered}
\sum_{k=0}^{m_{t}-1} f_{n}\left(X_{n}\left(t_{k}\right)\right)\left(L_{n}^{c}\left(t_{k+1}\right)-L_{n}^{c}\left(t_{k}\right)\right)-\int_{a}^{t} f(X(s)) d L^{c}(s) \\
=\sum_{k=0}^{m_{t}-1}\left(f_{n}\left(X_{n}\left(t_{k}\right)\right)-f\left(X\left(t_{k}\right)\right)\right)\left(L^{c}\left(t_{k+1}\right)-L^{c}\left(t_{k}\right)\right) \\
+\sum_{k=0}^{m_{t}-1} f_{n}\left(X_{n}\left(t_{k}\right)\right)\left(\left(L_{n}^{c}\left(t_{k+1}\right)-L_{n}^{c}\left(t_{k}\right)\right)-\left(L^{c}\left(t_{k+1}\right)-L^{c}\left(t_{k}\right)\right)\right) \\
\quad+\sum_{k=0}^{m_{t}-1} \int_{t_{k}}^{t_{k+1}}\left(f\left(X\left(t_{k}\right)\right)-f(X(s))\right) d L^{c}(s) \\
-\int_{a}^{t_{0}} f(X(s)) d L^{c}(s)=I_{1}+I_{2}+I_{3}-I_{4} .
\end{gathered}
$$

Since $f$ is Lipschitz continuous we have from (3.2)

$$
\left|I_{1}\right| \leq C / n+C \sum_{k=0}^{m_{t}-1}\left|X_{n}\left(t_{k}\right)-X\left(t_{k}\right)\right|\left|L^{c}\left(t_{k+1}\right)-L^{c}\left(t_{k}\right)\right| .
$$

By using the "summation by parts" formula we get for $I_{2}$

$$
\begin{gathered}
I_{2}=\sum_{k=1}^{m_{t}-1}\left(f_{n}\left(X_{n}\left(t_{k-1}\right)\right)-f_{n}\left(X_{n}\left(t_{k}\right)\right)\right)\left(L_{n}^{c}\left(t_{k}\right)-L^{c}\left(t_{k}\right)\right) \\
+f_{n}\left(X_{n}\left(t_{m_{t}-1}\right)\right)\left(L_{n}^{c}\left(t_{m_{t}}\right)-L^{c}\left(t_{m_{t}}\right)\right)-f_{n}\left(X_{n}\left(t_{0}\right)\right)\left(L_{n}^{c}\left(t_{0}\right)-L^{c}\left(t_{0}\right)\right) .
\end{gathered}
$$

It follows from Lemma 4.5, the Lipschitz continuity of $f$, and from the inequalities (3.1) and (5.2), that

$$
\left|I_{2}\right| \leq C\left(1+\left|X_{n}^{0}\left(\tau_{t}\right)\right|\right) \sup _{|u-v| \leq 1 / n} \mathrm{~V}_{u}^{v} L^{c} .
$$

Also, Lemma 4.5 implies the following estimation for $I_{3}$ :

$$
\left|I_{3}\right| \leq C h_{n}+C \sup _{|u-v| \leq h_{n}} \mathrm{~V}_{u}^{v} L^{c} .
$$

By using inequality (3.1) it easy to show that

$$
\left|I_{4}\right| \leq C \mathrm{~V}_{a}^{t_{0}} L^{c} \leq C \sup _{|u-v| \leq h_{n}} \mathrm{~V}_{u}^{v} L^{c} .
$$

The inequalities (5.3) - (5.6) imply the statement of the lemma.

Suppose that for any $t \in \mathbf{T}$ and $n \in \mathbb{N}$ the partition $0=\xi_{0}^{n}(t) \leq \xi_{1}^{n}(t) \leq \ldots \leq \xi_{p+2}^{n}(t)=1$, where $p$ depends on $n$, of the interval $[0,1]$ is given. Consider the recurrent sequence $\varphi_{k}^{n}(t)$, $t \in \mathbf{T}, k=0,1 \ldots p+2$ of the functions given by

$$
\left\{\begin{array}{l}
\varphi_{k+1}^{n}(t)=\varphi_{k}^{n}(t)+z\left(\varphi_{k}^{n}(t)\right)\left(\xi_{k+1}^{n}(t)-\xi_{k}^{n}(t)\right) \\
\varphi_{0}^{n}(t)=x
\end{array}\right.
$$


where $x \in \mathbb{R}$, and $z$ is a Lipschitz continuous function with constant $K$, such that

$$
|z(y)| \leq K(1+|y|), y \in \mathbb{R}
$$

Denote for all $u \in[0 ; 1], t \in \mathbf{T}$ and $n=1,2, \ldots$

$$
\sigma^{n}(u, t)= \begin{cases}\xi_{k}^{n}(t), & \text { if } \quad \xi_{k-1}^{n}(t)<u \leq \xi_{k}^{n}(t), \\ 0, & \text { if } \quad u=0 .\end{cases}
$$

and

$$
\phi^{n}(u, t)= \begin{cases}\varphi_{k}^{n}(t), & \text { if } \quad \xi_{k-1}^{n}(t)<u \leq \xi_{k}^{n}(t) \\ x, & \text { if } \quad u=0\end{cases}
$$

Then

$$
\phi^{n}(u, t)=x+\int_{[0 ; u)} z\left(\phi^{n}(s, t)\right) d_{s} \sigma^{n}(s, t)
$$

Lemma 5.3 Suppose that there exists a nondecreasing left continuous function $\sigma(u), u \in$ $[0 ; 1]$ such that

$$
\int_{\mathbf{T}}\left|\sigma^{n}(u, t)-\sigma(u)\right| d t \rightarrow 0
$$

as $n \rightarrow \infty$ for any continuity point $u$ of $\sigma$. Then $\sigma$ belongs to class $\mathbf{G}$ and

$$
\int_{\mathbf{T}}\left|\phi^{n}(u, t)-\phi(u)\right| d t \rightarrow 0
$$

as $n \rightarrow \infty$ for any continuity point $u$ of $\phi$, where $\phi(u)$ is a solution of the equation

$$
\phi(u)=x+\int_{[0, u)} z(\phi(s)) d \sigma(s) .
$$

Remark 5.4 Notice that $\phi(u)=\varphi(z, x, u, \mu)$, where $\varphi(z, x, u, \mu)$ is a solution of the equation (3.8) with measure $\mu$ generated by $\sigma$. Furthermore $\phi^{n}(u, t)=\varphi\left(z, x, u, \mu^{n}(t)\right)$, where $\varphi\left(z, x, u, \mu^{n}(t)\right)$ is a solution of the equation (3.8) with the measure $\mu^{n}(d u, t)$ generated by $\sigma^{n}(u, t)$.

Proof. From the definition of $\sigma^{n}$ we have for any $u \in[0 ; 1]$ that $\sigma^{n}(u, t) \geq u$ for all $t \in \mathbf{T}$. Hence $\sigma(u) \geq u$ for all $u \in[0 ; 1]$. Set $A=\{w \in[0 ; 1]: \sigma(w)=w\}$ and $B=\{w \in[0 ; 1]: \sigma(w)>w\}$. Let $u \in[0 ; 1]$ be a continuity point of $\sigma$ and $u \in B$. Then $\int_{\mathbf{T}}\left|\sigma^{n}(u, t)-d\right| d t \rightarrow 0$, where $d=\sigma(u)>u$. For any subsequence $\left\{n^{\prime}\right\}$ of $\{n\}$ we can choose a subsequence $\left\{n^{\prime \prime}\right\}$ of $\left\{n^{\prime}\right\}$ such that $\sigma^{n^{\prime \prime}}(u, t) \rightarrow d$ for almost all $t \in \mathbf{T}$. By Egorov's theorem (see, i.g., [15, Ch. 2, $\S 9$, Th. 2, p.55]) for any $\delta>0$ there exists a measurable set $\mathbf{T}_{\delta} \subset \mathbf{T}$ such that $\left|\mathbf{T} \backslash \mathbf{T}_{\delta}\right|<\delta$ and on the set $\mathbf{T}_{\delta}$ the sequence $\sigma^{n^{\prime \prime}}(u, t)$ converges uniformly to $d$. Here and in what follows $|D|$ denotes Lebesgue measure of the set $D$. Therefore for any $v \in(u, d)$ and large enough $n^{\prime \prime} \in \mathbb{N}$ we have $u<v<\sigma^{n^{\prime \prime}}(u, t)$ for all $t \in \mathbf{T}_{\delta}$. Hence $\sigma^{n^{\prime \prime}}(u, t)=\sigma^{n^{\prime \prime}}(v, t)$ for all $t \in \mathbf{T}_{\delta}$. Moreover

$$
\int_{\mathbf{T}}\left|\sigma^{n^{\prime \prime}}(v, t)-d\right| d t \leq \int_{\mathbf{T}_{\delta}}\left|\sigma^{n^{\prime \prime}}(u, t)-d\right| d t+2 \delta
$$


Letting $n \rightarrow \infty$ since $\delta$ is an arbitrary small we have $\int_{\mathbf{T}}\left|\sigma^{n^{\prime \prime}}(v, t)-d\right| d t \rightarrow 0$. It means that for each $v \in(u ; d)$ and any subsequence $\left\{n^{\prime}\right\}$ of $\{n\}$ we can choose a subsequence $\left\{n^{\prime \prime}\right\}$ of $\left\{n^{\prime}\right\}$ such that $\int_{\mathbf{T}}\left|\sigma^{n^{\prime \prime}}(v, t)-d\right| d t \rightarrow 0$. Hence $\int_{\mathbf{T}}\left|\sigma^{n}(v, t)-d\right| d t \rightarrow 0$ and $\sigma(v)=\sigma(u)=d>v$. Therefore $(u ; d) \subset B$.

It follows from the left-continuity of $\sigma$ that there exists $\xi>0$ such that for any $w \in$ $(u-\xi ; u)$ we have $d=\sigma(u) \geq \sigma(w)>u>w$. Hence $(u-\xi ; u) \subset B$. Therefore $(u-\xi ; d) \subset B$ and $B$ is an open set. Consequently we can write $B=\bigcup_{i \in I}\left(a_{i} ; b_{i}\right)$, where $\left(a_{i} ; b_{i}\right) \cap\left(a_{j} ; b_{j}\right)=\emptyset$ if $i \neq j$.

Let $x \in\left(a_{i} ; b_{i}\right)$ for some $i \in I$. If $\sigma(x)>b_{i}$ then $x<b_{i}<\sigma(x)$ and, as was shown above, $\sigma\left(b_{i}\right)=\sigma(x)>b_{i}$. Hence $b_{i} \in B$, but by definition $b_{i} \notin B$. Therefore $\sigma(x) \leq b_{i}$. If $d=\sigma(x)<b_{i}$, then $a_{i}<x<\sigma(x)=d<b_{i}$ and $\sigma(d)=d$, i.e. $d \notin B$. This contradiction shows that $\sigma(x)=b_{i}$. Since $\sigma(x)=x$ if $x \notin B$ then the function $\sigma$ belongs to class $\mathbf{G}$.

Suppose $\sigma$ has the form (3.10). For any $\epsilon>0$ there is a number $N \in \mathbb{N}$ such that $\sum_{i=N+1}^{\infty}\left(b_{i}-a_{i}\right)<\epsilon$. Consider the function

$$
\bar{\sigma}^{N}(u, t)=\left\{\begin{array}{lll}
\sigma(u), & \text { if } & u \notin \bigcup_{i=N+1}^{\infty}\left(a_{i} ; b_{i}\right] \\
u, & \text { if } & u \in \bigcup_{i=N+1}^{\infty}\left(a_{i} ; b_{i}\right]
\end{array}\right.
$$

It is evident that $\mathrm{V}_{0}^{1}\left|\sigma-\bar{\sigma}^{N}\right| \leq \epsilon$. Let $\bar{\phi}^{N}$ be a solution of the following equation:

$$
\bar{\phi}^{N}(u)=x+\int_{[0 ; u)} z\left(\bar{\phi}^{N}(s)\right) d \bar{\sigma}^{N}(s), u \in[0 ; 1] .
$$

Lipschitz continuity for $z$ and Lemma 4.3 imply

$$
\left|\bar{\phi}^{N}(u)-\phi(u)\right| \leq K \int_{[0 ; u)}\left|\bar{\phi}^{N}(s)-\phi(s)\right| d \sigma(s)+K_{1} \bigvee_{0}^{1}\left|\sigma-\bar{\sigma}^{N}\right|,
$$

where $K_{1}=(|x|+K) e^{K}$. Applying Lemma 4.1 we get

$$
\left|\bar{\phi}^{N}(u)-\phi(u)\right| \leq K_{1} \bigvee_{0}^{1}\left|\sigma-\bar{\sigma}^{N}\right| e^{K \sigma(u)} \leq K_{1} e^{K} \epsilon .
$$

Denote by $k_{i}, i=1,2, \ldots, N$ the number such that $\xi_{k_{i}}^{n}(t)<\left(a_{i}+b_{i}\right) / 2 \leq \xi_{k_{i}+1}^{n}(t)$. We assume that $a_{0}=b_{0}=0$ and $a_{i}=b_{i}=0, i=1,2, \ldots$ if $N=0$. Set $k_{0}=-1, k_{N+1}=p+3$ and $N_{k}=\max \left\{i: k_{i} \leq k\right\}, k=0,1, \ldots, p+2$. For any $n \in \mathbb{N}, l=0,1, \ldots, p+2$ and $t \in \mathbf{T}$ we define

$$
\widetilde{\xi}_{l}^{n}(t)= \begin{cases}a_{i}, & \text { if } \quad a_{i}<\xi_{l}^{n}(t) \leq \xi_{k_{i}}^{n}(t), \\ b_{i}, & \text { if } \quad b_{i}>\xi_{l}^{n}(t) \geq \xi_{k_{i}+1}^{n}(t), \\ \xi_{l}^{n}(t), & \text { in the other cases. }\end{cases}
$$

It follows from (5.7) that we can write $\varphi_{k+1}^{n}(t)$ in the following form:

$$
\begin{gathered}
\varphi_{k+1}^{n}(t)=x+\sum_{l=0}^{k} z\left(\varphi_{l}^{n}(t)\right)\left(\xi_{l+1}^{n}(t)-\xi_{l}^{n}(t)\right)=x+\sum_{i=1}^{N_{k}} z\left(\varphi_{k_{i}}^{n}(t)\right)\left(\xi_{k_{i}+1}^{n}(t)-\xi_{k_{i}}^{n}(t)\right) \\
+\sum_{i=1}^{N_{k}+1} \sum_{l=k_{i-1}+1}^{\left(k_{i}-1\right) \wedge k} z\left(\varphi_{l}^{n}(t)\right)\left(\xi_{l+1}^{n}(t)-\xi_{l}^{n}(t)\right) .
\end{gathered}
$$


From the equations (5.12) and (5.14) we have for any $n \in \mathbb{N}, k=0,1, \ldots, p+2$ and $t \in \mathbf{T}$

$$
\begin{aligned}
& \left|\varphi_{k+1}^{n}(t)-\bar{\phi}^{N}\left(\widetilde{\xi}_{k+1}^{n}(t)\right)\right| \leq \sum_{i=1}^{N_{k}}\left|z\left(\varphi_{k_{i}}^{n}(t)\right)\left(\xi_{k_{i}+1}^{n}(t)-\xi_{k_{i}}^{n}(t)\right)-z\left(\bar{\phi}^{N}\left(a_{i}\right)\right)\left(b_{i}-a_{i}\right)\right| \\
& +\sum_{i=1}^{N_{k}+1} \sum_{l=k_{i-1}+1}^{\left(k_{i}-1\right) \wedge k}\left|z\left(\varphi_{l}^{n}(t)\right)\left(\xi_{l+1}^{n}(t)-\xi_{l}^{n}(t)\right)-z\left(\bar{\phi}^{N}\left(\widetilde{\xi}_{l}^{n}(t)\right)\right)\left(\xi_{l+1}^{n}(t)-\xi_{l}^{n}(t)\right)\right| \\
& +\sum_{i=1}^{N_{k}}\left|\sum_{l=k_{i-1}+1}^{k_{i}-1} z\left(\bar{\phi}^{N}\left(\widetilde{\xi}_{l}^{n}(t)\right)\right)\left(\xi_{l+1}^{n}(t)-\xi_{l}^{n}(t)\right)-\int_{b_{i-1}}^{a_{i}} z\left(\bar{\phi}^{N}(s)\right) d s\right| \\
& +\left|\sum_{l=k_{N_{k}}+1}^{k} z\left(\bar{\phi}^{N}\left(\widetilde{\xi}_{l}^{n}(t)\right)\right)\left(\xi_{l+1}^{n}(t)-\xi_{l}^{n}(t)\right)-\int_{b_{N_{k}}}^{\tilde{\xi}_{k+1}^{n}(t)} z\left(\bar{\phi}^{N}(s)\right) d s\right| \\
& =\sum_{i=1}^{N_{k}} I_{1}^{i}+\sum_{i=1}^{N_{k}+1} I_{2}^{i}+\sum_{i=1}^{N_{k}} I_{3}^{i}+I_{4} .
\end{aligned}
$$

Consider $I_{3}^{i}, i=1,2, \ldots, N_{k}$. If $b_{i-1}=a_{i}$, then $\widetilde{\xi}_{l}^{n}(t)=a_{i}$ for $k_{i-1}+1 \leq l \leq k_{i}-1$ and from Lemma 4.3 and inequality (5.8) we have

$$
I_{3}^{i} \leq\left|\sum_{l=k_{i-1}+1}^{k_{i}-1} z\left(\bar{\phi}^{N}\left(a_{i}\right)\right)\left(\xi_{l+1}^{n}(t)-\xi_{l}^{n}(t)\right)\right| \leq K_{2}\left|b_{i-1}-\xi_{k_{i-1}+1}^{n}(t)\right|+K_{2}\left|a_{i}-\xi_{k_{i}}^{n}(t)\right|,
$$

where $K_{2}=K\left(1+(|x|+K) e^{K}\right)$.

If $b_{i-1}<a_{i}$, then $\widetilde{\xi}_{l}^{n}(t)=b_{i-1}$ for $\xi_{k_{i-1}+1}^{n}(t) \leq \xi_{l}^{n}(t)<\sigma^{n}\left(b_{i-1}, t\right)$ and $\widetilde{\xi}_{l}^{n}(t)=a_{i}$ for $\xi_{k_{i}}^{n}(t) \geq \xi_{l}^{n}(t) \geq \sigma^{n}\left(a_{i}, t\right)$. Hence

$$
I_{3}^{i} \leq K_{2}\left|b_{i-1}-\xi_{k_{i-1}+1}^{n}(t)\right|+K_{2}\left|a_{i}-\xi_{k_{i}}^{n}(t)\right|+\int_{b_{i-1}}^{a_{i}}\left|z\left(\bar{\phi}^{N}\left(\widetilde{\sigma}^{n}(s, t)\right)\right)-z\left(\bar{\phi}^{N}(s)\right)\right| d s,
$$

where $\widetilde{\sigma}^{n}(s, t)=\widetilde{\xi}_{l}^{n}(t)$ if $\xi_{l}^{n}(t)<s \leq \xi_{l+1}^{n}(t), l=0, \ldots, p+2$

Since $z$ is Lipschitz continuous with constant $K$, then Lemma 4.3 and inequalities (5.16) and (5.17) yield

$$
I_{3}^{i} \leq K_{2}\left|b_{i-1}-\xi_{k_{i-1}+1}^{n}(t)\right|+K_{2}\left|a_{i}-\xi_{k_{i}}^{n}(t)\right|+K K_{1} \int_{b_{i-1}}^{a_{i}}\left|\widetilde{\sigma}^{n}(s, t)-s\right| d s .
$$

Operating in the same way one can obtain the following estimation for $I_{4}$ :

$$
I_{4} \leq K_{2}\left|b_{N_{k}}-\xi_{k_{N_{k}}+1}^{n}(t)\right|+K K_{1} \int_{b_{N_{k}}}^{a_{N_{k}+1}}\left|\widetilde{\sigma}^{n}(s, t)-s\right| d s
$$

For $I_{1}^{i}$ by using Lemma 4.3 we have

$$
I_{1}^{i} \leq K_{2}\left|b_{i-1}-\xi_{k_{i-1}+1}^{n}(t)\right|+K_{2}\left|a_{i}-\xi_{k_{i}}^{n}(t)\right|+K\left|\varphi_{k_{i}}^{n}(t)-\bar{\phi}^{N}\left(a_{i}\right)\right|\left|\xi_{k_{i}+1}^{n}(t)-\xi_{k_{i}}^{n}(t)\right| .
$$


For $z$ Lipschitz continuous we get

$$
I_{2}^{i} \leq K \sum_{l=k_{i-1}+1}^{\left(k_{i}-1\right) \wedge k}\left|\varphi_{l}^{n}(t)-\bar{\phi}^{N}\left(\widetilde{\xi}_{l}^{n}(t)\right)\right|\left|\xi_{l+1}^{n}(t)-\xi_{l}^{n}(t)\right| .
$$

Collecting formulas (5.15), (5.18), (5.19), (5.20) and (5.21) we have

$$
\begin{gathered}
\left|\varphi_{k+1}^{n}(t)-\bar{\phi}^{N}\left(\widetilde{\xi}_{k+1}^{n}(t)\right)\right| \leq 2 K_{2} \sum_{i=1}^{N_{k}}\left(\left|b_{i}-\xi_{k_{i}+1}^{n}(t)\right|+\left|a_{i}-\xi_{k_{i}}^{n}(t)\right|\right) \\
+K K_{1} \sum_{i=1}^{N_{k}+1} \int_{b_{i-1}}^{a_{i}}\left|\widetilde{\sigma}^{n}(s, t)-s\right| d s+K \sum_{l=0}^{k}\left|\varphi_{l}^{n}(t)-\bar{\phi}^{N}\left(\widetilde{\xi}_{l}^{n}(t)\right)\right|\left|\xi_{l+1}^{n}(t)-\xi_{l}^{n}(t)\right| .
\end{gathered}
$$

Applying Lemma 4.2 to the inequality above we obtain

$$
\begin{gathered}
\left|\varphi_{k+1}^{n}(t)-\bar{\phi}^{N}\left(\widetilde{\xi}_{k+1}^{n}(t)\right)\right| \leq\left(2 K_{2} \sum_{i=1}^{N_{k}}\left(\left|b_{i}-\xi_{k_{i}+1}^{n}(t)\right|+\left|a_{i}-\xi_{k_{i}}^{n}(t)\right|\right)\right. \\
\left.+K K_{1} \sum_{i=1}^{N_{k}+1} \int_{b_{i-1}}^{a_{i}}\left|\widetilde{\sigma}^{n}(s, t)-s\right| d s\right) e^{K \xi_{k+1}^{n}(t)} .
\end{gathered}
$$

Fix an arbitrary continuity point $u \in[0 ; 1]$ of the function $\phi$ and let $\xi_{k}^{n}(t)$ be such that $\xi_{k}^{n}(t)<u \leq \xi_{k+1}^{n}(t)$. From the definition of $\phi^{n}(u, t)$ and inequalities (5.13) and (5.22) we have

$$
\begin{gathered}
\left|\phi^{n}(u, t)-\phi(u)\right| \leq\left|\varphi_{k+1}^{n}(t)-\bar{\phi}^{N}\left(\widetilde{\xi}_{k+1}^{n}(t)\right)\right|+\left|\bar{\phi}^{N}\left(\widetilde{\xi}_{k+1}^{n}(t)\right)-\phi\left(\widetilde{\xi}_{k+1}^{n}(t)\right)\right| \\
+\left|\phi\left(\widetilde{\xi}_{k+1}^{n}(t)\right)-\phi(u)\right| \leq 2 K_{2} e^{K} \sum_{i=1}^{N}\left(\left|b_{i}-\xi_{k_{i}+1}^{n}(t)\right|+\left|a_{i}-\xi_{k_{i}}^{n}(t)\right|\right) \\
+K K_{1} e^{K} \sum_{i=1}^{N} \int_{b_{i-1}}^{a_{i}}\left|\widetilde{\sigma}^{n}(s, t)-s\right| d s+K K_{1} e^{K} \int_{b_{N}}^{1}\left|\widetilde{\sigma}^{n}(s, t)-s\right| d s+K_{1} e^{K} \epsilon \\
+\int_{\left[u ; \tilde{\xi}_{k+1}^{n}(t)\right)}|z(\phi(s))| d \sigma(s) .
\end{gathered}
$$

Taking the integral on $t \in \mathbf{T}$ in both sides of the above inequality yields

$$
\begin{gathered}
\int_{\mathbf{T}}\left|\phi^{n}(u, t)-\phi(u)\right| d t \leq 2 K_{2} e^{K} \sum_{i=1}^{N}\left(\int_{\mathbf{T}}\left|b_{i}-\xi_{k_{i}+1}^{n}(t)\right| d t+\int_{\mathbf{T}}\left|a_{i}-\xi_{k_{i}}^{n}(t)\right| d t\right) \\
+K K_{1} e^{K} \int_{\mathbf{T}} \int_{[0,1] \backslash \bigcup_{i=1}^{\infty}\left(a_{i}, b_{i}\right]}\left|\widetilde{\sigma}^{n}(s, t)-s\right| d s d t \\
+K_{1}(1+K) e^{K} \epsilon|\mathbf{T}|+\int_{\mathbf{T}} \int_{\left[u ; \widetilde{\xi}_{k+1}^{n}(t)\right)}|z(\phi(s))| d \sigma(s) d t \\
=2 K_{2} e^{K} \sum_{i=1}^{N}\left(J_{1}^{i}+J_{2}^{i}\right)+K K_{1} e^{K} J_{3}+K_{1}(1+K) e^{K} \epsilon|\mathbf{T}|+J_{4} .
\end{gathered}
$$


If $u \in\left[a_{i} ; b_{i}\right]$ for some $i=1, \ldots, N$, then $\widetilde{\xi}_{k+1}^{n}(t)=a_{i}$ or $\widetilde{\xi}_{k+1}^{n}(t)=b_{i}$. Hence $J_{4}=0$.

If $u \notin \bigcup_{i=1}^{N}\left[a_{i} ; b_{i}\right]$, then $\widetilde{\xi}_{k+1}^{n}(t)=\xi_{k+1}^{n}(t)=\sigma^{n}(u, t)$. Therefore from Lemma 4.3 and inequality (5.8) we have if $u \notin \bigcup_{i=1}^{N}\left[a_{i} ; b_{i}\right]$

$$
J_{4} \leq K_{1} \int_{\mathbf{T}}\left|\sigma^{n}(u, t)-u\right| d t \leq K_{1} \int_{\mathbf{T}}\left|\sigma^{n}(u, t)-\sigma(u)\right| d t+K_{1}|\mathbf{T}| \epsilon .
$$

Consequently for continuity point $u$ of $\phi$ we get

$$
\limsup _{n \rightarrow \infty} J_{4} \leq K_{1}|\mathbf{T}| \epsilon .
$$

Since $\left(a_{i}+b_{i}\right) / 2, i=1, \ldots, N$ is a continuity point of $\sigma$, then

$$
\int_{\mathbf{T}}\left|\sigma^{n}\left(\left(a_{i}+b_{i}\right) / 2, t\right)-\sigma\left(\left(a_{i}+b_{i}\right) / 2\right)\right| d t \rightarrow 0
$$

But $\sigma\left(\left(a_{i}+b_{i}\right) / 2\right)=b_{i}$ and $\sigma^{n}\left(\left(a_{i}+b_{i}\right) / 2, t\right)=\xi_{k_{i}+1}^{n}(t)$. Hence

$$
\int_{\mathbf{T}}\left|b_{i}-\xi_{k_{i}+1}^{n}(t)\right| d t \rightarrow 0
$$

Therefore, for all $i=1, \ldots, N$

$$
\lim _{n \rightarrow \infty} J_{1}^{i}=0 .
$$

Consider $J_{2}^{i}$. For any $\delta>0$ set $A_{n}^{\delta, i}=\left\{t \in \mathbf{T}: \xi_{k_{i}}^{n}(t)>a_{i}+\delta\right\}$. Then for $\delta<\left(b_{i}-a_{i}\right) / 2$ we have that $v=a_{i}+\delta<\left(a_{i}+b_{i}\right) / 2, \sigma(v)=b_{i}$ and $\left(a_{i}+b_{i}\right) / 2>\xi_{k_{i}}^{n}(t) \geq \sigma^{n}(v, t)$ for all $t \in A_{n}^{\delta, i}$. Hence, since $v$ is a continuity point of $\sigma$

$$
\left|A_{n}^{\delta, i}\right|\left(b_{i}-a_{i}\right) / 2 \leq \int_{A_{n}^{\delta, i}}\left|\xi_{k_{i}}^{n}(t)-b_{i}\right| d t \leq \int_{\mathbf{T}}\left|\sigma^{n}(v, t)-\sigma(v)\right| d t \rightarrow 0
$$

as $n \rightarrow \infty$. Thus $\left|A_{n}^{\delta, i}\right| \rightarrow 0$ for all $\delta<\left(b_{i}-a_{i}\right) / 2$ and $i=1, \ldots, N$.

Set $B_{n}^{\delta, i}=\left\{t \in \mathbf{T}: \xi_{k_{i}}^{n}(t)<a_{i}-\delta\right\}$. Then for $w=a_{i}-\delta$ we have $\sigma(w) \leq a_{i}$ and $\sigma^{n}(w, t)=\xi_{k_{i}+1}^{n}(t) \geq\left(a_{i}+b_{i}\right) / 2$ for $t \in B_{n}^{\delta, i}$. Hence, if $w$ is a continuity point of $\sigma$, then

$$
\left|B_{n}^{\delta, i}\right|\left(b_{i}-a_{i}\right) / 2 \leq \int_{B_{n}^{\delta, i}}\left|\sigma^{n}(w, t)-\sigma(w)\right| d t \leq \int_{\mathbf{T}}\left|\sigma^{n}(w, t)-\sigma(w)\right| d t \rightarrow 0
$$

as $n \rightarrow \infty$. Therefore $\left|B_{n}^{\delta, i}\right| \rightarrow 0$ for all $i=1, \ldots, N$ and almost all $\delta>0$.

Thus for all $i=1, \ldots, N$ and almost all $\left(b_{i}-a_{i}\right) / 2>\delta>0$

$$
\limsup _{n \rightarrow \infty} \int_{\mathbf{T}}\left|\xi_{k_{i}}^{n}(t)-a_{i}\right| d t=\limsup _{n \rightarrow \infty} \int_{\mathbf{T} \backslash\left(A_{n}^{\delta, i} \cup B_{n}^{\delta, i}\right)}\left|\xi_{k_{i}}^{n}(t)-a_{i}\right| d t \leq \delta|\mathbf{T}| .
$$

Since $\delta$ can be arbitrary small then for all $i=1, \ldots, N$

$$
\lim _{n \rightarrow \infty} J_{2}^{i}=\lim _{n \rightarrow \infty} \int_{\mathbf{T}}\left|\xi_{k_{i}}^{n}(t)-a_{i}\right| d t=0 .
$$


Consider $J_{3}$. For any $s \in[0 ; 1] \backslash \bigcup_{i=1}^{\infty}\left(a_{i}, b_{i}\right]$ and $\delta>0$ denote by $C_{n}^{\delta}(s)$ the following subset of $\mathbf{T}: C_{n}^{\delta}(s)=\left\{t \in \mathbf{T}: \widetilde{\sigma}^{n}(s, t)<s-\delta\right\}$. For all $v=s-\delta$ we have $\sigma^{n}(v, t)=$ $\sigma^{n}(s, t) \geq s=\sigma(s)>\sigma(v) \geq v$ for $t \in C_{n}^{\delta}(s)$. Hence, if $v$ is a continuity point of $\sigma$, then

$$
(s-\sigma(v))\left|C_{n}^{\delta}(s)\right| \leq \int_{C_{n}^{\delta}(s)}\left|\sigma^{n}(v, t)-\sigma(v)\right| d t \leq \int_{\mathbf{T}}\left|\sigma^{n}(v, t)-\sigma(v)\right| d t \rightarrow 0 .
$$

Thus $\left|C_{n}^{\delta}(s)\right| \rightarrow 0$ as $n \rightarrow \infty$ for $s \notin \bigcup_{i=1}^{\infty}\left(a_{i}, b_{i}\right]$ and almost all $\delta>0$. Therefore, since $\tilde{\sigma}^{n}(s, t)<s$ we have

$$
\limsup _{n \rightarrow \infty} \int_{\mathbf{T}}\left|\widetilde{\sigma}^{n}(s, t)-s\right| d t=\limsup _{n \rightarrow \infty} \int_{\mathbf{T} \backslash C_{n}^{\delta}(s)}\left|\widetilde{\sigma}^{n}(s, t)-s\right| d t \leq \delta|\mathbf{T}|
$$

Hence

$$
\lim _{n \rightarrow \infty} \int_{\mathbf{T}}\left|\widetilde{\sigma}^{n}(s, t)-s\right| d t=0 .
$$

It follows from Lebesgue's theorem that

$$
\lim _{n \rightarrow \infty} J_{3}=\lim _{n \rightarrow \infty} \int_{[0,1] \backslash \bigcup_{i=1}^{\infty}\left(a_{i}, b_{i}\right]} \int_{\mathbf{T}}\left|\widetilde{\sigma}^{n}(s, t)-s\right| d t d s=0 .
$$

Thus from equalities (5.23), (5.25), (5.26), (5.27) and (5.24) we obtain

$$
\limsup _{n \rightarrow \infty} \int_{\mathbf{T}}\left|\phi^{n}(u, t)-\phi(u)\right| d t \leq K_{1}\left((1+K) e^{K}+1\right) \epsilon|\mathbf{T}|
$$

for any continuity point $u \in[0 ; 1]$ of $\phi$ and for all $\epsilon>0$. Hence

$$
\lim _{n \rightarrow \infty} \int_{\mathbf{T}}\left|\phi^{n}(u, t)-\phi(u)\right| d t=0,
$$

which completes the proof of the lemma.

Denote by $j_{i}, i=1, \ldots$ the number such that $t_{j_{i}} \leq \zeta_{i}-1 / n<t_{j_{i}+1}$. Set $\xi_{k}^{n, i}(t)=$ $F_{n}\left(\zeta_{i}-t_{j_{i}+k}\right), i=1, \ldots, t \in \mathbf{T}, n \in \mathbb{N}$ and $k=0,1, \ldots, p+2$, where $p=\left[1 /\left(n h_{n}\right)\right]$ (brackets denote the integer part of the number). Notice that $\xi_{k}^{n, i}(t)$ depends on $t \in \mathbf{T}$, since $t_{j_{i}+k}$ depends on $t$. Denote by $\varphi_{k}^{n, i}(z, x, t)$ the sequence which is defined by the formula (5.7) for $\xi_{k}^{n}(t)=\xi_{k}^{n, i}(t)$. Let $\sigma_{i}^{n}(u, t)$ be the sequence of functions which is given by the formula (5.9) with $\xi_{n}(t)=\xi_{k}^{n, i}(t)$.

The following lemma from [16] (see also [17]) will give the necessary and sufficient conditions for $\sigma_{i}^{n}(u, t)$ to satisfy the statement of Lemma 5.3.

Lemma 5.5 ([16]) Suppose that $F_{n}\left(F_{n}^{-1}(u)-\delta h_{n}\right) \rightarrow \sigma(u)$ as $n \rightarrow \infty$ and $h_{n} \rightarrow 0$ for all $\delta \in(0 ; 1)$ and all continuity points $u \in[0 ; 1]$ of $\sigma$. Then

$$
\int_{\mathbf{T}}\left|\sigma_{i}^{n}(u, t)-\sigma(u)\right| d t \rightarrow 0
$$

as $n \rightarrow \infty$ and $h_{n} \rightarrow 0$ for $i=1,2, \ldots$ and all continuity points $u \in[0 ; 1]$ of $\sigma$. 
Conversely, if there exists function $\sigma(u, t), u \in[0 ; 1], t \in \mathbf{T}$ such that $\sigma(u, t) \in L^{1}(\mathbf{T})$ for any $u \in[0 ; 1], \sigma(u, t)$ is left continuous and nondecreasing on $u$ for any $t \in \mathbf{T}$, and for each continuous function $z:[0 ; 1] \rightarrow \mathbb{R}$ we have that for all $i=1,2, \ldots$

$$
\int_{\mathbf{T}}\left|\int_{0}^{1} z(u) \sigma_{i}^{n}(d u, t)-\int_{0}^{1} z(u) \sigma_{i}(d u, t)\right| d t,
$$

Then $\sigma_{i}(u, t)$ does not depend on $i$ and $t$, i.e. $\sigma_{i}(u, t)=\sigma(u)$ and $F_{n}\left(F_{n}^{-1}(u)-\delta h_{n}\right) \rightarrow \sigma(u)$ for all continuity points $u \in[0 ; 1]$ of $\sigma$ and for any $\delta \in(0 ; 1)$.

Lemma 5.6 Let $f$ and $g$ be a Lipschitz continuous function satisfying (3.1). For any $\varepsilon>0$ the following inequality is true for large enough $n$ :

$$
\begin{gathered}
\left.\mid \sum_{k=0}^{m_{t}-1} f_{n}\left(X_{n}\left(t_{k}\right)\right)\right)\left(L_{n}^{d}\left(t_{k+1}\right)-L_{n}^{d}\left(t_{k}\right)\right)-\sum_{\zeta_{i} \leq t}\left(\varphi_{p+2}^{n, i}\left(\Delta L\left(\zeta_{i}\right) f, X_{n}\left(t_{j_{i}}\right), t\right)-X_{n}\left(t_{j_{i}}\right)\right) \mid \\
\leq C\left(1+\left|X_{n}^{0}\left(\tau_{t}\right)\right|\right)\left(\sup _{|u-v| \leq 2 / n+h_{n}} \mathrm{~V}_{u}^{v} L^{c}+1 / n+h_{n}+\varepsilon\right) .
\end{gathered}
$$

Proof. Let $N \in \mathbb{N}$ be from the representation (5.1). Then for large enough $n$ we have that

$$
1 / n+h_{n} \leq \min _{0 \leq k<j \leq N}\left|\zeta_{k}-\zeta_{j}\right|
$$

where $\zeta_{0}=a$.

If the inequality (5.28) holds, then $L_{n}^{\leq N}(t)=L^{\leq N}(t)$ for all $t \notin \bigcup_{i=1}^{N}\left(\zeta_{i}-1 / n, \zeta_{i}\right)$. Moreover, $\left(t_{j_{i}}, t_{j_{i}+p+2}\right] \bigcap\left(t_{j_{r}}, t_{j_{r}+p+2}\right]=\emptyset$, if $i \neq r$. Hence by definition of $L_{n}^{\leq N}$ we get

$$
\begin{gathered}
L_{n}^{\leq N}\left(t_{j_{i}+l+1}\right)-L_{n}^{\leq N}\left(t_{j_{i}+l}\right)=\Delta L\left(\zeta_{i}\right)\left(F_{n}\left(\zeta_{i}-t_{j_{i}+l+1}\right)-F_{n}\left(\zeta_{i}-t_{j_{i}+l}\right)\right) \\
=\Delta L\left(\zeta_{i}\right)\left(\xi_{l+1}^{n, i}(t)-\xi_{l}^{n, i}(t)\right)
\end{gathered}
$$

for all $l=0,1 \ldots p+1$ and $i=1,2 \ldots N$.

By using this equality we have

$$
\begin{gathered}
\left.\left.\sum_{k=0}^{m_{t}-1} f_{n}\left(X_{n}\left(t_{k}\right)\right)\right)\left(L_{n}^{d}\left(t_{k+1}\right)-L_{n}^{d}\left(t_{k}\right)\right)=\sum_{k=0}^{m_{t}-1} f_{n}\left(X_{n}\left(t_{k}\right)\right)\right)\left(L_{n}^{>N}\left(t_{k+1}\right)-L_{n}^{>N}\left(t_{k}\right)\right) \\
+\sum_{\zeta_{i} \leq t} \sum_{l=0}^{p+1} f_{n}\left(X_{n}\left(t_{j_{i}+l}\right)\right) \Delta L^{\leq N}\left(\zeta_{i}\right)\left(\xi_{l+1}^{n, i}(t)-\xi_{l}^{n, i}(t)\right) .
\end{gathered}
$$

Thus

$$
\begin{aligned}
\left.\mid \sum_{k=0}^{m_{t}-1} f_{n}\left(X_{n}\left(t_{k}\right)\right)\right) & \left(L_{n}^{d}\left(t_{k+1}\right)-L_{n}^{d}\left(t_{k}\right)\right)-\sum_{\zeta_{i} \leq t}\left(\varphi_{p+2}^{n, i}\left(\Delta L\left(\zeta_{i}\right) f, X_{n}\left(t_{j_{i}}\right), t\right)-X_{n}\left(t_{j_{i}}\right)\right) \mid \\
& \left.\leq \mid \sum_{k=0}^{m_{t}-1} f_{n}\left(X_{n}\left(t_{k}\right)\right)\right)\left(L_{n}^{>N}\left(t_{k+1}\right)-L_{n}^{>N}\left(t_{k}\right)\right) \mid
\end{aligned}
$$




$$
\begin{gathered}
+\left|\sum_{\zeta_{i} \leq t}\left(\varphi_{p+2}^{n, i}\left(\Delta L^{>N}\left(\zeta_{i}\right) f, X_{n}\left(t_{j_{i}}\right), t\right)-X_{n}\left(t_{j_{i}}\right)\right)\right| \\
+\mid \sum_{\zeta_{i} \leq t} \sum_{l=0}^{p+1} f_{n}\left(X_{n}\left(t_{j_{i}+l}\right)\right) \Delta L^{\leq N}\left(\zeta_{i}\right)\left(\xi_{l+1}^{n, i}(t)-\xi_{l}^{n, i}(t)\right) \\
-\sum_{\zeta_{i} \leq t}\left(\varphi_{p+2}^{n, i}\left(\Delta L^{\leq N}\left(\zeta_{i}\right) f, X_{n}\left(t_{j_{i}}\right), t\right)-X_{n}\left(t_{j_{i}}\right)\right) \mid=I_{1}+I_{2}+I_{3} .
\end{gathered}
$$

By the formulas (3.1) and (5.1) we have from Remark 5.4 and Lemma 4.3

$$
I_{1}+I_{2} \leq C\left(1+\left|X_{n}^{0}\left(\tau_{t}\right)\right|\right) \varepsilon
$$

Consider $I_{3}$. By using representations (3.4), (5.1) and the definition of $\varphi_{k}^{n, i}$ we have for all $i=1, \ldots, N, n \in \mathbb{N}, t \in \mathbf{T}$, and $l=1, \ldots, p+2$

$$
\begin{aligned}
& \mid X_{n}\left(t_{j_{i}+l}\right)-\varphi_{l}^{n, i}\left(\Delta L^{\leq N}\left(\zeta_{i}\right) f, X_{n}\left(t_{j_{i}}\right), t\right)\left|\leq \sum_{k=0}^{l-1}\right| g_{n}\left(X_{n}\left(t_{j_{i}+k}\right)\right) \mid h_{n} \\
&+\left|\sum_{k=0}^{l-1} f_{n}\left(X_{n}\left(t_{j_{i}+k}\right)\right)\left(L_{n}^{c}\left(t_{j_{i}+k+1}\right)-L_{n}^{c}\left(t_{j_{i}+k}\right)\right)\right| \\
&+\left|\sum_{k=0}^{l-1} f_{n}\left(X_{n}\left(t_{j_{i}+k}\right)\right)\left(L_{n}^{>N}\left(t_{j_{i}+k+1}\right)-L_{n}^{>N}\left(t_{j_{i}+k}\right)\right)\right| \\
&+\left|\Delta L^{\leq N}\left(\zeta_{i}\right)\right| \sum_{k=0}^{l-1}\left|f_{n}\left(X_{n}\left(t_{j_{i}+k}\right)\right)-f\left(\varphi_{k}^{n, i}\left(\Delta L^{\leq N}\left(\zeta_{i}\right) f, X_{n}\left(t_{j_{i}}\right), t\right)\right)\right|\left(\xi_{l+1}^{n, i}(t)-\xi_{l}^{n, i}(t)\right) .
\end{aligned}
$$

Since $f$ and $g$ are Lipschitz continuous satisfying the inequality (3.1) we get from Lemma 4.2 and representation $(5.1)$

$$
\left|X_{n}\left(t_{j_{i}+l}\right)-\varphi_{l}^{n, i}\left(\Delta L^{\leq N}\left(\zeta_{i}\right) f, X_{n}\left(t_{j_{i}}\right), t\right)\right| \leq C\left(1+\left|X_{n}^{0}\left(\tau_{t}\right)\right|\right)\left(h_{n}+1 / n+\sup _{|u-v| \leq 2 / n+h_{n}} \mathrm{~V}_{u}^{v} L^{c}+\varepsilon\right),
$$

for any $l=1, \ldots, p+2$.

Hence, from the inequality above and the definition of $\varphi_{k}^{n, i}$ we obtain

$$
\begin{gathered}
I_{3} \leq \sum_{\zeta_{i} \leq t} \sum_{l=0}^{p+1}\left|f_{n}\left(X_{n}\left(t_{j_{i}+l}\right)\right)-f\left(\varphi_{l}^{n, i}\left(\Delta L^{\leq N}\left(\zeta_{i}\right) f, X_{n}\left(t_{j_{i}}\right), t\right)\right) \| \Delta L^{\leq N}\left(\zeta_{i}\right)\right|\left(\xi_{l+1}^{n, i}(t)-\xi_{l}^{n, i}(t)\right) \\
\leq C\left(1+\left|X_{n}^{0}\left(\tau_{t}\right)\right|\right)\left(h_{n}+1 / n+\sup _{|u-v| \leq 2 / n+h_{n}} \mathrm{~V}_{u}^{v} L^{c}+\varepsilon\right) .
\end{gathered}
$$

Inequalities (5.29) and (5.30) imply the statement of the lemma. 


\section{Proof of the main theorem}

In this section we will prove Theorem 3.4.

Proof of TheOREM 3.4. Let $F_{n}\left(F_{n}^{-1}(u)-\delta h_{n}\right) \rightarrow \sigma(u)$ as $n \rightarrow \infty$ and as $h_{n} \rightarrow 0$ for all $\delta \in(0 ; 1)$ and for all continuity points $u \in[0 ; 1]$ of $\sigma$.

Let us show that if $X_{n}$ is a solution of the equation (3.3) and if $X$ is a solution of the equation (3.7) with the measure $\mu(d u)$ generated by the function $\sigma(u)$, then $X_{n}$ converges to $X$ in $L^{1}(\mathbf{T})$.

Fix an arbitrary $\varepsilon>0$. Let $L=L^{c}+L^{\leq N}+L^{>N}$, where $L^{c}$ is a continuous part of $L$, and $L^{\leq N}$ and $L^{>N}$ are as stated in the equality (5.1). By using this representation and formula (3.4) we have

$$
\begin{gathered}
X_{n}(t)-X(t)=\left(X_{n}^{0}\left(\tau_{t}\right)-x^{0}\right)+\left(\sum_{k=0}^{m_{t}-1} g_{n}\left(X_{n}\left(t_{k}\right)\right) h_{n}-\int_{a}^{t} g(X(s)) d s\right) \\
+\left(\sum_{k=0}^{m_{t}-1} f_{n}\left(X_{n}\left(t_{k}\right)\right)\left(L_{n}^{c}\left(t_{k+1}\right)-L_{n}^{c}\left(t_{k}\right)\right)-\int_{a}^{t} f(X(s)) d L^{c}(s)\right) \\
\left.+\left(\sum_{k=0}^{m_{t}-1} f_{n}\left(X_{n}\left(t_{k}\right)\right)\right)\left(L_{n}^{d}\left(t_{k+1}\right)-L_{n}^{d}\left(t_{k}\right)\right)-\sum_{a<s \leq t}(\varphi(\Delta L(s) f, X(s-), 1)-X(s-))\right) \\
=\left(X_{n}^{0}\left(\tau_{t}\right)-x^{0}\right)+H_{1}+H_{2}+H_{3} .
\end{gathered}
$$

Lemma 5.1 implies

$$
\left|H_{1}\right| \leq C h_{n}+C / n+C h_{n} \sum_{k=0}^{m_{t}-1}\left|X_{n}\left(t_{k}\right)-X\left(t_{k}\right)\right| .
$$

The following inequality is a consequence of Lemma 5.2.

$$
\begin{aligned}
\left|H_{2}\right| \leq C h_{n}+C / n+C\left(1+\left|X_{n}^{0}\left(\tau_{t}\right)\right|\right) \sup _{|u-v| \leq h_{n}+1 / n} \mathrm{~V}_{u}^{v} L^{c} \\
+C \sum_{k=0}^{m_{t}-1}\left|X_{n}\left(t_{k}\right)-X\left(t_{k}\right)\right|\left|L^{c}\left(t_{k+1}\right)-L^{c}\left(t_{k}\right)\right| .
\end{aligned}
$$

For $H_{3}$ by using the same notations $\varphi_{k}^{n, i}$ and $j_{i}$ as in Lemma 5.6 we get

$$
\begin{aligned}
& \left.\left|H_{3}\right| \leq \mid \sum_{k=0}^{m_{t}-1} f_{n}\left(X_{n}\left(t_{k}\right)\right)\right)\left(L_{n}^{d}\left(t_{k+1}\right)-L_{n}^{d}\left(t_{k}\right)\right)-\sum_{\zeta_{i} \leq t}\left(\varphi_{p+2}^{n, i}\left(\Delta L\left(\zeta_{i}\right) f, X_{n}\left(t_{j_{i}}\right), t\right)-X_{n}\left(t_{j_{i}}\right)\right) \mid \\
& +\left|\sum_{\zeta_{i} \leq t}\left(\varphi_{p+2}^{n, i}\left(\Delta L^{>N}\left(\zeta_{i}\right) f, X_{n}\left(t_{j_{i}}\right), t\right)-X_{n}\left(t_{j_{i}}\right)\right)\right| \\
& +\left|\sum_{\zeta_{i} \leq t}\left(\varphi_{p+2}^{n, i}\left(\left(\Delta L^{\leq N}\left(\zeta_{i}\right) f, X_{n}\left(t_{j_{i}}\right), t\right)-X_{n}\left(t_{j_{i}}\right)\right)-\left(\varphi_{p+2}^{n, i}\left(\Delta L^{\leq N}\left(\zeta_{i}\right) f, X\left(t_{j_{i}}\right), t\right)-X\left(t_{j_{i}}\right)\right)\right)\right|
\end{aligned}
$$




$$
\begin{gathered}
+\left|\sum_{\zeta_{i} \leq t}\left(\left(\varphi_{p+2}^{n, i}\left(\Delta L^{\leq N}\left(\zeta_{i}\right) f, X\left(t_{j_{i}}\right), t\right)-X\left(t_{j_{i}}\right)\right)-\left(\varphi_{p+2}^{n, i}\left(\Delta L^{\leq N}\left(\zeta_{i}\right) f, X\left(\zeta_{i}-\right), t\right)-X\left(\zeta_{i}-\right)\right)\right)\right| \\
+\left|\sum_{\zeta_{i} \leq t}\left(\left(\varphi_{p+2}^{n, i}\left(\Delta L^{\leq N}\left(\zeta_{i}\right) f, X\left(\zeta_{i}-\right), t\right)-X\left(\zeta_{i}-\right)\right)-\left(\varphi\left(\Delta L^{\leq N}\left(\zeta_{i}\right) f, X\left(\zeta_{i}-\right), 1\right)-X\left(\zeta_{i}-\right)\right)\right)\right| \\
+\left|\sum_{a<s \leq t}\left(\varphi\left(\Delta L^{>N}(s) f, X(s-), 1\right)-X(s-)\right)\right|=S_{1}+S_{2}+S_{3}+S_{4}+S_{5}+S_{6} .
\end{gathered}
$$

From Lemma 5.6 we deduce the following estimation for $S_{1}$ :

$$
S_{1} \leq C\left(1+\left|X_{n}^{0}\left(\tau_{t}\right)\right|\right)\left(\sup _{|u-v| \leq 2 / n+h_{n}} \mathrm{~V}_{u}^{v} L^{c}+1 / n+h_{n}+\varepsilon\right) .
$$

The definition of $L^{>N}$ and Lemmas 4.3 and 4.5 imply

$$
S_{2} \leq C\left(1+\left|X_{n}^{0}\left(\tau_{t}\right)\right|\right) \varepsilon
$$

and

$$
S_{6} \leq C \varepsilon
$$

Applying Lemma 4.3 to $S_{3}$ we obtain

$$
S_{3} \leq C \sum_{i=1}^{N}\left|\Delta L\left(\zeta_{i}\right)\right|\left|X_{n}\left(t_{j_{i}}\right)-X\left(t_{j_{i}}\right)\right|
$$

Operating in the same way we have

$$
S_{4} \leq C \sum_{i=1}^{N}\left|\Delta L\left(\zeta_{i}\right)\right|\left|X\left(t_{j_{i}}\right)-X\left(\zeta_{i}-\right)\right|
$$

It follows from Lemma 4.5 that for all $i=1, \ldots N$

$$
\left|X\left(t_{j_{i}}\right)-X\left(\zeta_{i}-\right)\right| \leq C\left|t_{j_{i}}-\zeta_{i}\right|+C \mathrm{~V}_{t_{j_{i}}}^{\zeta_{i}-} L .
$$

By the definition of $t_{j_{i}}$ we have $\left|t_{j_{i}}-\zeta_{i}\right| \leq h_{n}+1 / n$. Moreover from the inequality (5.28) we deduce that $\mathrm{V}_{t_{j_{i}}}^{\zeta_{i}-} L \leq \mathrm{V}_{t_{j_{i}}}^{\zeta_{i}} L^{c}+\varepsilon$ for all $i=1, \ldots N$. Hence

$$
S_{4} \leq C \sum_{i=1}^{N}\left|\Delta L\left(\zeta_{i}\right)\right|\left(\left|t_{j_{i}}-\zeta_{i}\right|+C \mathrm{~V}_{t_{j_{i}}}^{\zeta_{i}} L^{c}+\varepsilon\right) \leq C h_{n}+C / n+C \sup _{|u-v| \leq h_{n}+1 / n} \mathrm{~V}_{u}^{v} L^{c}+C \varepsilon
$$

Consider $S_{5}$.

$$
S_{5} \leq \sum_{i=1}^{N}\left|\varphi_{p+2}^{n, i}\left(\Delta L\left(\zeta_{i}\right) f, X\left(\zeta_{i}-\right), t\right)-\varphi\left(\Delta L\left(\zeta_{i}\right) f, X\left(\zeta_{i}-\right), 1\right)\right|=\sum_{i=1}^{N} z_{n}^{i, N}(t) .
$$

The inequalities (6.4)-(6.9) imply the following estimation for $H_{3}$ :

$$
\left|H_{3}\right| \leq C\left(1+\left|X_{n}^{0}\left(\tau_{t}\right)\right|\right)\left(\sup _{|u-v| \leq 2 / n+h_{n}} \mathrm{~V}_{u}^{v} L^{c}+1 / n+h_{n}+\varepsilon\right)
$$




$$
+C \sum_{i=1}^{N}\left|\Delta L\left(\zeta_{i}\right)\right|\left|X_{n}\left(t_{j_{i}}\right)-X\left(t_{j_{i}}\right)\right|+\sum_{i=1}^{N} z_{n}^{i, N}(t) .
$$

Hence, from the expressions (6.2), (6.3) and (6.10) we have

$$
\begin{aligned}
\left|X_{n}(t)-X(t)\right| \leq & \left|X_{n}^{0}\left(\tau_{t}\right)-x^{0}\right|+C\left(1+\left|X_{n}^{0}\left(\tau_{t}\right)\right|\right)\left(\sup _{|u-v| \leq 2 / n+h_{n}} \mathrm{~V}_{u}^{v} L^{c}+1 / n+h_{n}+\varepsilon\right) \\
& +C \sum_{k=0}^{m_{t}-1}\left|X_{n}\left(t_{k}\right)-X\left(t_{k}\right)\right|\left(\left|L^{c}\left(t_{k+1}\right)-L^{c}\left(t_{k}\right)\right|+h_{n}\right) \\
& +C \sum_{i=1}^{N}\left|\Delta L\left(\zeta_{i}\right)\right|\left|X_{n}\left(t_{j_{i}}\right)-X\left(t_{j_{i}}\right)\right|+\sum_{i=1}^{N} z_{n}^{i, N}(t) .
\end{aligned}
$$

Applying Lemma 4.2 to the inequality (6.11) and taking the integral over $\mathbf{T}$ we obtain

$$
\begin{gathered}
\int_{\mathbf{T}}\left|X_{n}(t)-X(t)\right| d t \leq C \int_{\mathbf{T}}\left|X_{n}^{0}\left(\tau_{t}\right)-x^{0}\right| d t+C \sum_{i=1}^{N} \int_{\mathbf{T}} z_{n}^{i, N}(t) d t \\
+C\left(|\mathbf{T}|+\int_{\mathbf{T}}\left|X_{n}^{0}\left(\tau_{t}\right)\right| d t\right)\left(\sup _{|u-v| \leq 2 / n+h_{n}} \mathrm{~V}_{u}^{v} L^{c}+1 / n+h_{n}+\varepsilon\right) .
\end{gathered}
$$

From the conditions of Theorem 3.4 we have

$$
\int_{\mathbf{T}}\left|X_{n}^{0}\left(\tau_{t}\right)-x^{0}\right| d t \rightarrow 0
$$

Hence $\int_{\mathbf{T}}\left|X_{n}^{0}\left(\tau_{t}\right)\right| d t \leq C$ for all $n \in \mathbb{N}$.

Since $\mathrm{V}_{a}^{t} L^{c}, t \in \mathbf{T}$ is a continuous function, then it is a uniformly continuous on $\mathbf{T}$, thus if $n \rightarrow \infty$ and $h_{n} \rightarrow 0$ then $\sup _{|u-v| \leq 2 / n+h_{n}} \mathrm{~V}_{u}^{v} L^{c} \rightarrow 0$.

Recall that $\varphi_{k}^{n, i}(z, x, t)$ is given by the expression $(5.7)$ with $\xi_{n}(t)=\xi_{k}^{n, i}(t)=F_{n}\left(\zeta_{i}-t_{j_{i}+k}\right)$, $i=1, \ldots, t \in \mathbf{T}, n \in \mathbb{N}$ and $k=0,1, \ldots, p+2$, where $p=\left[1 /\left(n h_{n}\right)\right]$. As stated before Lemma 5.5 denote by $\sigma_{i}^{n}(u, t)$ the sequence of the functions which is given by the formula (5.9) with $\xi_{n}(t)=\xi_{k}^{n, i}(t)=F_{n}\left(\zeta_{i}-t_{j_{i}+k}\right)$. It follows from Lemma 5.3 that $\int_{\mathbf{T}} z_{n}^{i, N}(t) d t \rightarrow 0$ as $n \rightarrow \infty$ for any $i=1, \ldots, N$, and $N \in \mathbb{N}$ if

$$
\int_{\mathbf{T}}\left|\sigma_{i}^{n}(u, t)-\sigma(u)\right| d t \rightarrow 0
$$

for any continuity point $u \in[0 ; 1]$ of the function $\sigma$. But from Lemma 5.5 we have that $(6.13)$ holds if and only if $F_{n}\left(F_{n}^{-1}(u)-\delta h_{n}\right) \rightarrow \sigma(u)$ as $n \rightarrow \infty$ and as $h_{n} \rightarrow 0$ for all $\delta \in(0 ; 1)$ and all continuity points $u \in[0 ; 1]$ of $\sigma$. Therefore $\int_{\mathbf{T}} z_{n}^{i, N}(t) d t \rightarrow 0$ and letting $n \rightarrow \infty$ and $h_{n} \rightarrow 0$ in the inequality $(6.12)$ we obtain

$$
\int_{\mathbf{T}}\left|X_{n}(t)-X(t)\right| d t \rightarrow 0
$$

which completes the proof of Theorem 3.4. 
Proof of Corollary 3.5. Let $\delta$-sequence $\rho_{n}$ be of the simplest type, i.e. $\rho_{n}(u)=n \rho(n u)$, where $\rho \in C^{\infty}(\mathbb{R}), \rho \geq 0$, supp $\rho \subseteq[0 ; 1]$ and $\int_{0}^{1} \rho(u) d u=1$. Then $F_{n}\left(F_{n}^{-1}(u)-\delta h_{n}\right)=$ $F\left(F^{-1}(u)-\delta n h_{n}\right)$, where $F(t)=\int_{t}^{1} \rho(s) d s$, and $F^{-1}(s)=\sup \{u: F(u)=s\}$. Hence the sequence $F_{n}\left(F_{n}^{-1}(u)-\delta h_{n}\right)$ converges weakly for any $\delta \in(0 ; 1)$, and the limit does not depend on $\delta$ if and only if either $1 / n=o\left(h_{n}\right)$ or $h_{n}=o(1 / n)$.

If $1 / n=o\left(h_{n}\right)$ then $F_{n}\left(F_{n}^{-1}(u)-\delta h_{n}\right)=F\left(F^{-1}(u)-\delta n h_{n}\right) \rightarrow H(u)$, where $H(u)=0$, if $u=0$, and $H(u)=1$ if $u>0$.

If $h_{n}=o(1 / n)$ then $F_{n}\left(F_{n}^{-1}(u)-\delta h_{n}\right)=F\left(F^{-1}(u)-\delta n h_{n}\right) \rightarrow u$, for all $u \in[0 ; 1]$.

Applying Theorem 3.4 we have the statement of Corollary 3.5.

Proof of Corollary 3.6. Since $L$ is a continuous then in the equality (6.1) we have that $H_{3}=0$. Hence $z_{n}^{i, N}(t)=0$ and we can take $\varepsilon=0$ in the inequality (6.12). Letting then $n \rightarrow \infty$ and $h_{n} \rightarrow \infty$ in the formula (6.12) we obtain the statement of Corollary 3.6.

The statement of Remark 3.7 follows from the inequality (6.11) by using the analogous arguments as before the inequality (6.12).

In conclusion let us note that Theorem 3.4 says that the sequence of solutions of the equation (3.3) converges to the solution of the equation (3.7) with the measure $\mu$ generated by the function from the class G. On the other hand the equation (3.7) has a solution for any measure $\mu$. In order to prove a similar theorem for another class of measure $\mu$ one has to consider another representative $L_{n}$ of the generalized function $\widetilde{L}$. For example it is possible to take $L_{n}$ as a convolution with the $\delta$-sequence $\rho_{n}$, where $\rho_{n}$ can take negative values.

\section{Acknowledgments}

I would like to thank Bernt Øksendal for his encouragement and interest, Paul Kettler for the attentive reading and the Department of Mathematics, University of Oslo, for its warm hospitality. This work was supported by INTAS grant 03-55-1861.

\section{References}

[1] Antosik, P., Ligeza, J.: Products of measures and functions of finite variations. Generalized functions and operational calculus (Proc. Conf., Varna, 1975), pp. 20-26, Bulgar. Acad. Sci., Sofia, 1979.

[2] Das, P. C., Sharma, R. R.: Existence and stability of measure differential equations. Czech. Math. J. 22 (1972), no 1, 145-158.

[3] Filippov, A. F.: Differential equations with discontinuous right hand sides. Mathematics and its Applications (Soviet Series), 18. Kluwer Academic Publishers Group, Dordrecht, 1988 .

[4] Ligeza, J.: On generalized solutions of some differential nonlinear equations of order $n$ Ann. Polon. Math. 31 (1975), no. 2, 115-120.

[5] Ligeza, J.: The existence and uniqueness of some systems of nonlinear differential equations. Časopis Pěst. Mat. 102 (1977), no. 1, 30-36. 
[6] Pandit, S. G., Deo, S. G.: Differential systems involving impulses. Lect. Notes Math, Berlin 1982. V. 954.

[7] Zavalishchin, S. T., Sesekin, A. N.: Dynamic impulse systems. Theory and applications. Mathematics and its Applications, 394. Kluwer Academic Publishers Group, Dordrecht, 1997.

[8] Egorov, Yu. V.: A contribution to the theory of generalized functions. Russian Math. Surveys 45 (1990), 1-49.

[9] Lazakovich, N. V., Stashulenok, S. P., Yufereva, I. V.: Stochastic differential equations in the algebra of generalized random processes. Differential Equations 31 (1995), no. 12, 2056-2058.

[10] Lazakovich, N. V.; Yablonski, O. L.: On the approximation of solutions of a class of stochastic equations. Siberian Math. J. 42 (2001), no. 1, 75-90.

[11] Lazakovich, N. V., Yablonski, A. L.: On the approximation of the solutions of stochastic equations with $\theta$-integrals. Stochastics and Stochastics Reports 76 (2004), no. 2, 135145.

[12] Lazakovich, N. V.: Stochastic differentials in the algebra of generalized random processes. (Russian) Dokl. Akad. Nauk Belarusi 38 (1994), no. 5, pp. 23-27.

[13] Groh, J.: A nonlinear Volterra-Stieltjes integral equation and a Gronwall inequality in one dimension. Illinis J. Math. 24 (1980), no. 2, 244-263.

[14] Constales, D.: Sharp bounds for the discrete analogue of a Gronwall-type inequality. Acta Math. Hungar. 80 (1998), no. 4, 325-334.

[15] Kolmogorov, A. N., Fomin, S. V.: Measure, Lebesgue integrals, and Hilbert space. Academic Press, New York-London 1961.

[16] Yablonski, O. L.: Stochastic integrals for jump random processes. (Russian) Dokl. Nats. Akad. Nauk Belarusi 46 (2002), no. 1, 42-46.

[17] Yablonski, O. L.: Stochastic integrals for random processes with independent increments. (Russian) Dokl. Nats. Akad. Nauk Belarusi 47 (2003), no. 3, 10-13 